\title{
Nuclear protein in testis midline carcinoma in a Turkish boy: a case report
}

\author{
Büşra Yaprak Bayrak ${ }^{1, *}$, Demir Kürşat Yildiz ${ }^{1 \odot}$, Uğur Demirsoy $^{2 \oplus}$, Funda Çorapçioğlu ${ }^{2}$, \\ Yonca Anik ${ }^{3 \odot}$, Christopher A. French ${ }^{4 \odot}$
}

\section{Abstract}

Background: Nuclear protein in testis (NUT) midline carcinoma (NMC) is an undifferentiated carcinoma, usually localized to the midline and presenting a translocation in the gene for bromodomain containing protein 4 . Here, we report a rare case of NMC in an 8-year-old Turkish boy.

Case report: There were masses in the lung, liver, and iliac wing representing metastases. Abdominal lymph node sampling revealed epithelial tumor infiltration with cellular pleomorphism. Immunohistochemistry was strongly positive for cytokeratin and epithelial membrane antigen protein. Because of undifferentiated carcinoma morphology, the tumor was considered to be a NMC. Immunoreactivity with antibodies to NUT and the presence of NUT clarified by fluorescence in situ hybridization (FISH) supported the diagnosis. Despite initial response to chemotherapy, the patient died 7 months after the diagnosis.

Conclusions: Immunoreactivity for NUT antibodies along with a dual-color FISH and karyotype analysis was suggestive for diagnosis of NMC. In differential diagnosis of undifferentiated carcinomas that occur particularly at midline localization, NMC should be considered.

Keywords: carcinoma; immunohistochemistry; in situ hybridization, fluorescence; nuclear protein in testis, human; Turkey

Nuclear protein in testis (NUT) midline carcinoma (NMC), also known as NUT carcinoma, is a poorly differentiated carcinoma characterized by translocations on chromosomes 15 and $19[\mathrm{t}(15 ; 19)(\mathrm{q} 14 ; \mathrm{p} 13.1)][1]$. This genetic aberration occurs as a result of the fusion between the gene for NUT midline carcinoma family member 1 (NUTM1) on chromosome 15 and the gene for the bromodomain-containing protein 4 (BRD4) on chromosome 19. The BRD4-NUT fusion oncogene, or the less frequently seen $B R D 3-N U T$ fusion oncogene, leads to the global hypoacetylation and suppression of the transcriptional gene required for differentiation [1, 2]. Members of the BRDNUT fusion protein family block epithelial differentiation and cell proliferation $[2,3]$. NMC is a rare disease recently identified, with an unknown prevalence due to previous lack of available testing methods and limited clinical awareness. Here, we present a rare case of NMC in an 8-year-old Turkish boy diagnosed through histopathology, immunohistochemistry, and fluorescence in situ hybridization (FISH).

*Correspondence to: Büşra Yaprak Bayrak, Department of Pathology, Faculty of Medicine, Kocaeli University, Kocaeli 41380, Turkey, e-mail: busra.yaprakbayrak@kocaeli.edu.tr 'Department of Pathology, Faculty of Medicine, Kocaeli University, Kocaeli 41380, Turkey ${ }^{2}$ Department of Pediatric Oncology, Faculty of Medicine, Kocaeli University, Kocaeli 41380, Turkey

${ }^{3}$ Department of Radiology, Faculty of Medicine, Kocaeli University, Kocaeli 41380, Turkey

${ }^{4}$ Department of Pathology, Harvard Medical School, Brigham and Women's Hospital, Boston, MA 02115, USA

Ә Open Access. ๑ 2020 Bayrak et al., published by Sciendo. (c) BY-NC-ND This work is licensed under the Creative Commons Attribution NonCommercial-NoDerivatives 4.0 License. 


\section{Case report}

An 8-year-old Turkish boy presented at our hospital with complaints of cough, sweating, and loss of appetite during the previous 2 months. On physical examination, his skin was found to be mildly pale; his sclera was anemic. He had hepatomegaly, and his left cervical lymph nodes were palpable. Auscultation of the lungs revealed rales. A low level of hemoglobin, leukocytosis, and high levels of lactate dehydrogenase and $\alpha$-fetoprotein were remarkable.

In computed tomography (CT) of the chest, infiltration and atelectasis of the right lung lower lobe were detected, and multiple lymph nodes in the thorax (the right upper and lower paratracheal, subcarinal, precarinal, right hilar, and para-aortic areas) were observed, the largest at $3 \mathrm{~cm}$ diameter. Abdominal magnetic resonance (MR) imaging revealed several metastatic masses in the liver and multiple abdominal lymph nodes, the largest at $2 \mathrm{~cm}$ diameter. Fluorodeoxyglucose $\left({ }^{18} \mathrm{~F}\right)\left(\left[{ }^{18} \mathrm{~F}\right] \mathrm{FDG}\right)$ positron emission tomography (PET)-CT revealed additional metastatic lesions in the left iliac bone.

As a clinicoradiological outcome, the diagnosis was non-Hodgkin lymphoma (Burkitt lymphoma). Biopsies were sampled from the bone marrow and the abdominal lymph node. However, the bone marrow biopsy was unremarkable. Histopathology of the para-aortic lymph node showed tumor metastasis. The tumor was in the form of undifferentiated cell clusters constituted from randomly distributed nests in the desmoplastic stroma. Tumor cells had evident amphophilic cytoplasm, an irregular nuclear membrane, fine chromatin, and prominent nucleoli. Mitotic figures and apoptotic bodies were frequent (Figure 1). Poorly differentiated squamous cell carcinoma (SCC), lymphoma, melanoma, germ cell tumors, and primitive neuroectodermal tumor (PNET)/Ewing sarcoma were included in the differential diagnosis.

A wide-range immunohistochemical panel was applied on $3 \mu \mathrm{m}$ sections from paraffin blocks using a fully automated system using the following antibodies: pan-keratin antibody (clone AE1/AE3; Ventana Medical Systems, cat. No. 2595, research resource identifier (RRID): AB_2335932), epithelial membrane antigen antibody (clone E29; Ventana Medical Systems, cat. No. 790-4463, RRID: AB_2335986), CD 30 antibody (clone Ber-H83; Ventana Medical Systems, cat. No. 790-2926, RRID: AB_2336012), CD 117 antibody (clone ACK2; Ventana Medical Systems, cat. No. 790-2951, RRID: AB_2335973), CD 45 (leukocyte common antigen) antibody (clone F10-89-4; Ventana Medical Systems, cat. No. 790-2930, RRID: AB_2336014), CD 2 antibody (clone EP222; Ventana Medical Systems, cat. No. 760-4377, RRID: AB_2336006), CD 20 antibody (clone UMAB58; Ventana Medical Systems, cat. No. 760-2531, RRID: AB_2335956),
CD 10 antibody (clone 56C6; Ventana Medical Systems, cat. No. 790-4506, RRID: AB_2336021), CD 34 antibody (clone QBEnd/10; Ventana Medical Systems, cat. No. 790-2927,
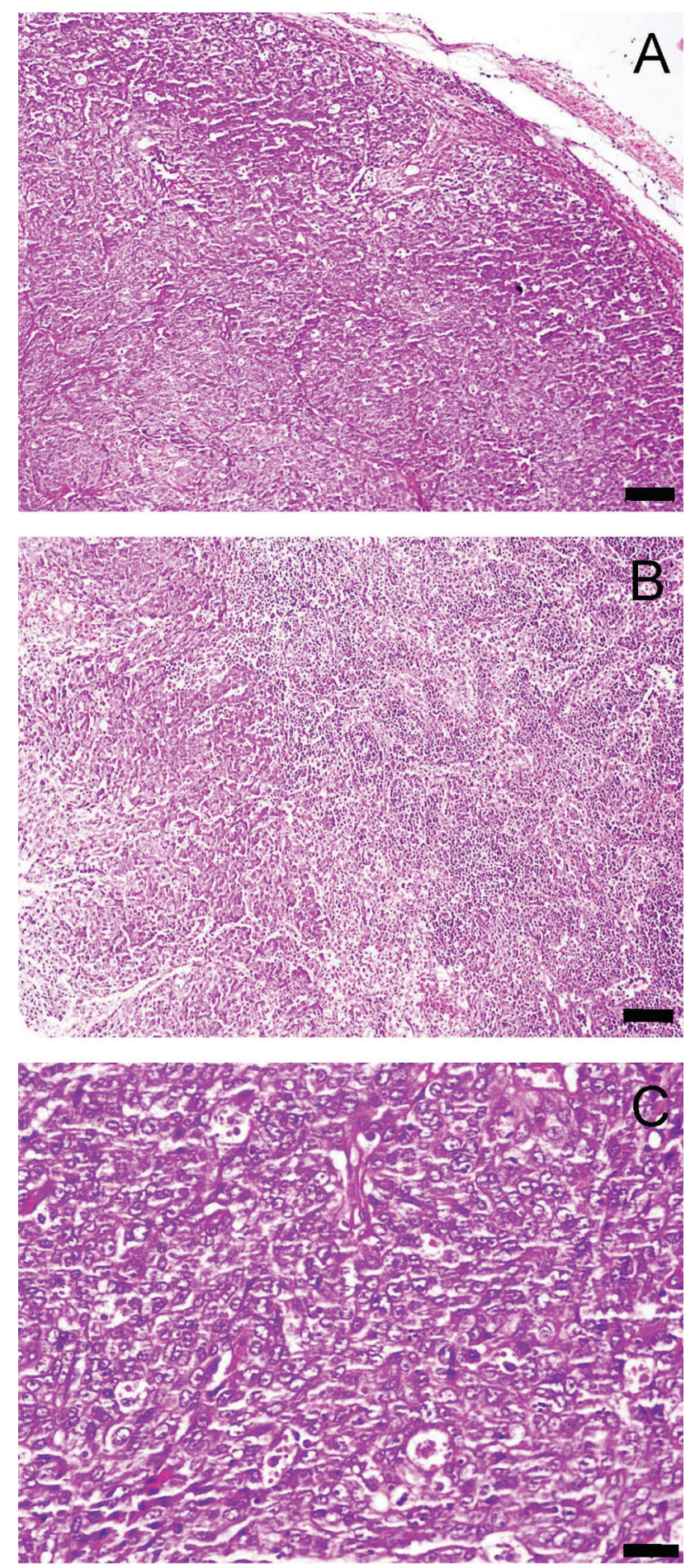

Figure 1. Histopathology of an abdominal lymph node derived from the patient with nuclear protein in testis (NUT) midline carcinoma. Undifferentiated tumor cells infiltrating into the lymph node are prominent. Hematoxylin and eosin staining. (A) $100 \times$ (scale bar $100 \mu \mathrm{m}$ ); (B) 100x (scale bar $100 \mu \mathrm{m}$ ); and (C) 400× (scale bar $25 \mu \mathrm{m}$ ). 
RRID: AB_2336013), CD 117 (cKit) antibody (clone EP10; Ventana Medical Systems, cat. No. 790-2951, RRID: AB 2335973), BCL-2 antibody (clone bcl-2; Ventana Medical Systems, cat. No. 2693, RRID: AB_2335938), BCL-6 antibody (clone GI191E; Ventana Medical Systems, cat. No. 7604241, RRID: AB_2335965), placental alkaline phosphatase antibody (clone NB10, Cell Marque, cat. No. 760-2664, RRID: AB_2335649), synaptophysin antibody (clone SY38; Ventana Medical Systems, cat. No. 790-4407, RRID: AB_2336016), chromogranin antibody (clone LK2H10; Ventana Medical Systems, cat. No. 760-2519, RRID: AB_2335955), CD 99 antibody (clone PCB1; Ventana Medical Systems, cat. No. 790-4452, RRID: AB_2336019), desmin antibody (clone 10H7D2; Ventana Medical Systems, cat. No. 2513, RRID: AB_2335926), Ki-67 antibody (clone MM1; Ventana Medical Systems, cat. No. 2520, RRID: AB_2335929), and anti-NUT antibody (clone C52B1; Cell Signaling Technology, cat. No. 3625, RRID: AB_2066833). An ultraView Universal DAB Detection Kit (Ventana Medical Systems, cat. No. 760-500) was used as an indirect, biotin-free system for detecting primary antibodies. The kit is intended to identify targets by immunohistochemistry using Meyer's hematoxylin as the counterstain. Interpretation of the immunohistochemistry was based on published data demonstrating distinctive granular (punctate or dusty) nuclear immunoreactivity present in $>50 \%$ of neoplastic cell nuclei [4]. Positive and negative controls were compared. Normal testicular tissue was used as a positive control for the NUT immunohistochemistry. The tumor cells had an intense positive reaction for pan-keratin antibody and epithelial membrane antigen (Figure 2); negative staining for CD 30, CD 117, CD 45, CD 2, CD 20, CD 10, CD 34, BCL-2, BCL-6, placental alkaline phosphatase, synaptophysin, chromogranin, CD 99, and desmin. The Ki-67 proliferation index was $70 \%$.

Because of the negative staining for a wide range of antibodies, the tumor was considered not to be differentiated into any of the common types of malignant tumors. Therefore, the preliminary diagnosis was "undifferentiated malignant epithelial tumor". Undifferentiated carcinoma morphology led to the consideration of NMC diagnosis. An expert pathologist (author CAF) reported the patient as having poorly differentiated carcinoma consistent with "NUT midline carcinoma" because of the positive immunoreactivity with antibodies against NUT (Figure 3), and the diagnosis was confirmed using FISH to detect gene translocation involving the NUT locus. FISH was performed on sections using the ZytoLight SPEC NUT Dual Color Break Apart Probe (ZytoVision, Bremerhaven, Germany) with standard protocols according to the manufacturer's instructions. The probes for NUT split-apart were NUT
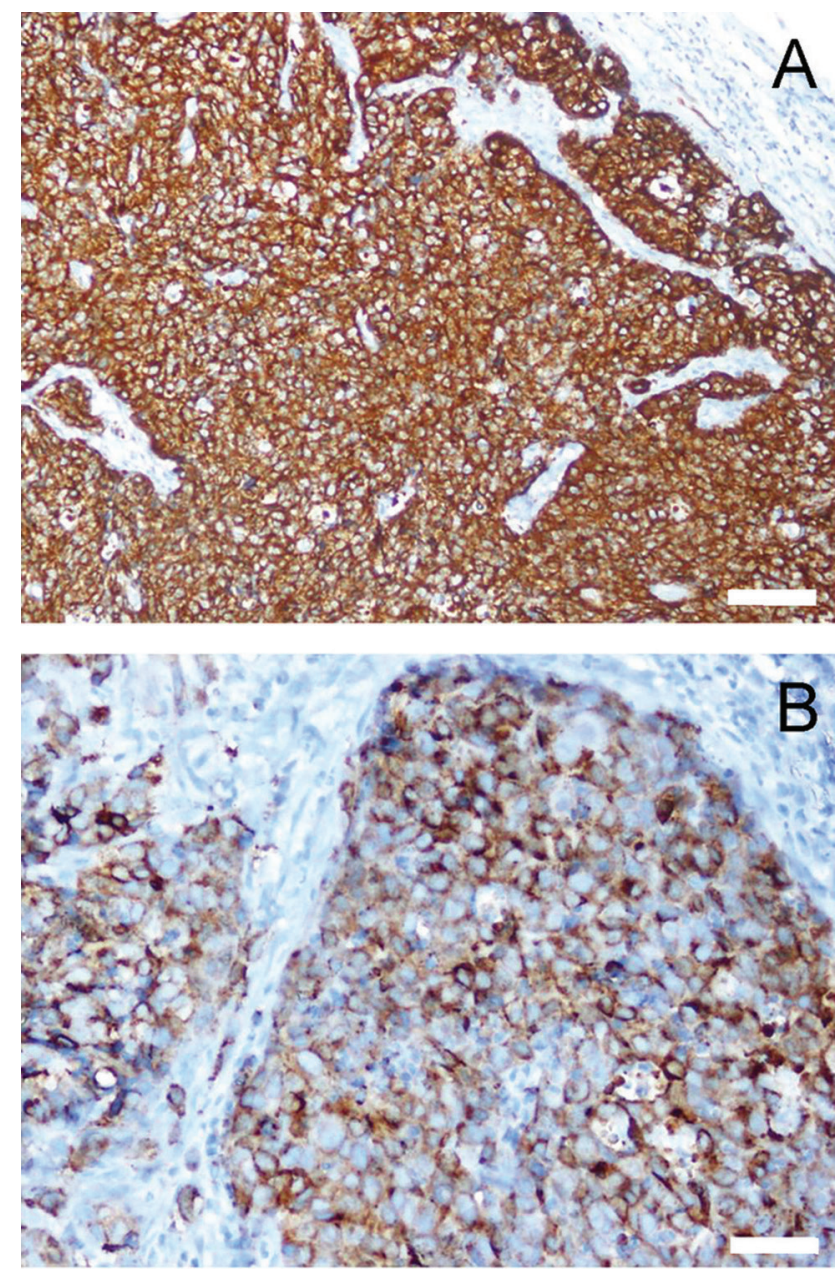

Figure 2. Micrograph images of an abdominal lymph node section immunohistochemistry derived from the patient with nuclear protein in testis (NUT) midline carcinoma. Immunoreactivity is indicated by the dark-brown staining from oxidized diaminobenzidine chromogen used in the method. The blue counterstain is Mayer's hematoxylin. A. Positive cytoplasmic immunoreactivity for cytokeratin (AE1/AE3), $200 \times$ (scale bar $50 \mu \mathrm{m}$ ). B. Positive cytoplasmic immunoreactivity for epithelial membrane antigen, $200 \times$ (scale bar $50 \mu \mathrm{m}$ ).

$5^{\prime}$ centromeric probes, namely, RP11-368L15 and RP111084A12 (biotin labeled, red), and NUT 3' telomeric probes, namely, RP11-1H8 and RP11-64o3 (digoxigenin labeled, green). The probes for BRD4 split-apart were BRD4 5' centromeric probes, namely, RP11-207i16 and RP11-3055m5 (biotin labeled, red), and BRD4 3' telomeric probes, namely, RP11-319O10 and RP11-681D10 (digoxigenin labeled, green). The presence of 2 pairs of fused green and orange signals was considered a normal finding, while the translocation-positive nuclei indicated 1 fused orange/green signal and 1 separate orange and green signal. In 4 different areas of each tumor, 200 nuclei were counted. We considered $80 \%$ positive interpretable nuclei as positive for a rearrangement. 

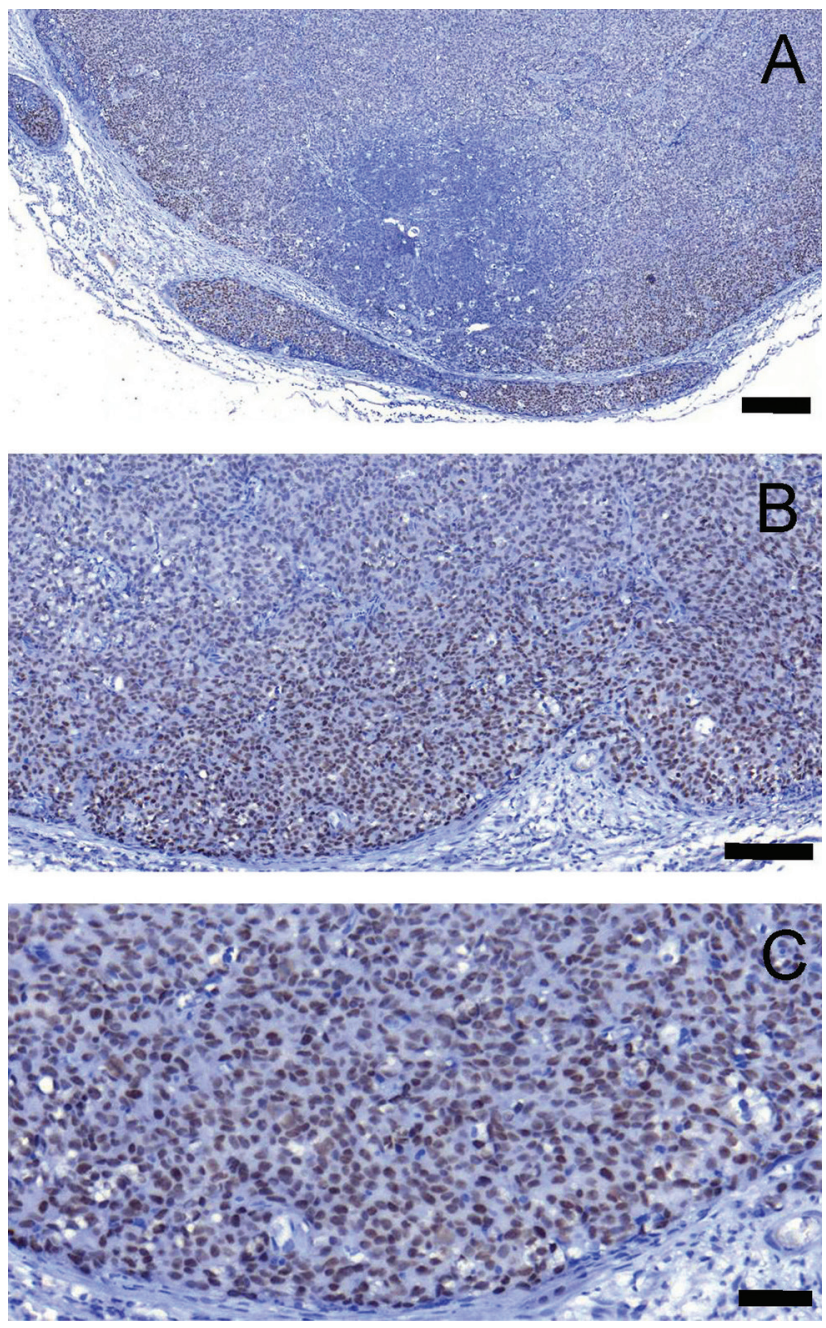

Figure 3. Positive nuclear immunoreactivity for nuclear protein in testis (NUT) in an abdominal lymph node derived from the patient with NUT midline carcinoma is indicated by the dark-brown staining of tumoral nuclei from oxidized diaminobenzidine chromogen used in the method. The blue counterstain is Meyer's hematoxylin, which stained nontumoral nuclei dark blue. Some cytoplasmic staining is also seen in lighter blue due to the long incubation time with the hematoxylin. (A) $40 \times$ (scale bar $200 \mu \mathrm{m}$ ), (B) 100× (scale bar $50 \mu \mathrm{m}$ ), and (C) $200 \times$ (scale bar $20 \mu \mathrm{m}$ ).

The results of FISH indicated $B R D 4$ rearrangement, consistent with FISH findings of $B R D 4-N U T$ fusion.

Initial chemotherapy involved dexamethasone $\left(5 \mathrm{mg} / \mathrm{m}^{2}\right.$ for 2 days and $10 \mathrm{mg} / \mathrm{m}^{2}$ for 3 days) and cyclophosphamide $\left(200 \mathrm{mg} / \mathrm{m}^{2}\right.$ for 2 days, according to the "Vorphase treatment" of non-Hodgkin lymphoma-Berlin-FrankfurtMünster [NHL-BFM]-2012 treatment protocol). After the biopsy, undifferentiated malignant cells were observed; so, ICE (ifosfamide, carboplatin, and etoposide) chemotherapy (ifosfamide $1800 \mathrm{mg} / \mathrm{m}^{2}$ on days $1-3$, etoposide $150 \mathrm{mg} / \mathrm{m}^{2}$ on days $1-3$, and carboplatin $500 \mathrm{mg} / \mathrm{m}^{2}$ on day 3) was initiated. Despite an initial partial response to ICE chemotherapy, the patient frequently presented at our emergency room. During the treatment, respiratory distress and difficulty in swallowing developed; so, the chemotherapeutic regime had to be revised. The patient also received radiotherapy (36 Gy radiotherapy in 18 fractions, as applied to the para-aortic and pelvic lymph nodes).

During the most recent follow-up, CT revealed the progressive nature of the tumor compared with the imaging 7 months earlier. There were increased mediastinal lymph nodes, mediastinal shift to the left, total atelectasis of the right lung, and bilateral pleural effusion. Metastatic masses were remarkable in the right pleura, the diaphragm, the humerus, the sternum, the vertebrae, and the right kidney. Despite all efforts, the patient died 7 months after the initial diagnosis. The father of the patient provided written consent for the publication of this case report and any accompanying information and images. This case report was exempted from formal review by our institutional ethics committee.

\section{Discussion}

Here, we describe a rare case of NMC, which is generally a fatal tumor, genetically identified as an undifferentiated carcinoma with partial SCC morphology. NMC was first reported as an intrathoracic highly malignant carcinoma by and Kees et al. [5] and Kubonishi et al. [6] in 1991. NMC is typically located in the midline and usually seen in the upper respiratory tract, the digestive tract, and the mediastinum [3]. However, it has also been reported in parathyroid glands, salivary glands, pancreas, and iliac bone [7-12]. Initially regarded as a pediatric tumor, cases now have been identified in patients across a wide range of ages ( $0-78$ years) among both male and female patients equally $[7,8]$. Prasad et al. reported 5 pediatric cases of NUT carcinoma, all of which were in patients who presented with a midline head and neck mass. Despite an aggressive multimodality treatment, only 1 patient survived [13]. Our patient also died at the end of 7 months after diagnosis despite aggressive chemoradiotherapy.

Karyotyping or FISH should be performed for a definitive diagnosis [3]. Unlike many cases of SCC, NUT has a complex karyotype, characterized by the presence of a single reciprocal translocation $t(15 ; 19)(q 14 ; p 13.1)$. These genetic aberrations cause the fusion of the 19P 13.1 chromosome from the BET family genes BRD4 and NUT on chromosome $15 \mathrm{q} 14$ [3]. Lemelle et al. [14] presented a case series in patients with a median age of 18.1 years (range 12.3-49.7 years). Half of the patients were initially 
misdiagnosed. Specific NUT antibody test was positive, and BRD4-NUT rearrangements were detected in all cases [14]. In a study by Zhou et al. analyzing 5 cases of NUT midline carcinoma, 2 cases were found to be localized in the lung, 1 in the larynx, 1 in the maxillary gingiva, and 1 in the orbital cavity [15]. NUT staining indicated strong diffuse nuclear staining in tumor cells, and FISH established the presence of NUT gene translocation in these cases. Approximately $70 \%$ of NMC cases present with a translocation of $B R D 4$, resulting in a roughly $6.4 \mathrm{~kb} B R D 4-N U T$ fusion gene. In the remaining $30 \%$ of NMC cases, NUT fuses with $B R D 3$ or other unknown genes $[2,16]$. In our patient, FISH results revealed a BRD4 rearrangement including BRD4-NUT fusion, and this was consistent with NMC.

Histopathology typically shows 2 types of tumor cell populations: poorly differentiated carcinoma and well-differentiated squamous cell islands with focal keratinization [7]. According to some reports, the tumor may contain trabecular and solid islands with an infiltrating margin and cord-like growth pattern or undifferentiated cell clusters arising from randomly distributed nests in the desmoplastic stroma [8]. On occasions, these tumors appear as clusters of small rounded cells with interstitial neutrophil infiltration [15].

According to World Health Organization characterization, NUT carcinomas commonly and characteristically demonstrate abrupt foci of keratinization [9]. This may include squamous eddies, single cell keratinization, intracellular bridges, cystic changes, necrosis, cholesterol granulomas, and psammoma-like bodies [10]. However, our patient had none of these features, so it was difficult to differentiate the diagnosis of NMC. Immunohistochemistry and FISH helped us to approach the exact diagnosis, suggesting a unique diagnostic view of NMC.

Histologically, NUT carcinoma is an undifferentiated or poorly differentiated carcinoma, marked by the constant expression of epithelial markers such as pan-cytokeratins (KL1 and AE1-AE3) on immunohistochemistry, and positive immunoreactivity for anti-cytokeratin antibodies, as well as epithelial membrane antigen, $\mathrm{p} 63$, and $\mathrm{p} 40$, which are the usual findings, and should raise the suspicion of NUT carcinoma in young individuals with a midline tumor [11]. Based on the nonspecific initial clinical presentation and histological features, an easy-to-use immunohistochemical tool is helpful to guide the diagnosis: diffuse and strong nuclear NUT immunoreactivity, revealing the probable presence of the NUT protein in significant quantities at the nuclear site, can confirm the diagnosis [11].

Differential diagnosis of NMC includes diagnostic challenges of undifferentiated carcinomas, poorly differentiated SCC, pediatric small blue round cell tumors (such as
PNET, rhabdomyosarcoma, and desmoplastic small round cell tumor), melanoma, olfactory neuroblastoma, high-grade hematologic malignancy, endocrine carcinomas, and sinonasal undifferentiated carcinoma [9]. Nuclear positivity of $>50 \%$ for anti-NUT antibody with FISH allows the diagnosis of NUT carcinoma with $100 \%$ specificity, suggesting that positivity with p63 and NUT antibody is diagnostic for NMC [11]. The other methods of diagnosis are dual-color FISH to fix NUT and BRD4 or showing translocations by karyotype analysis $\mathrm{t}(15 ; 19)$. Our FISH analysis proved the $B R D 4$ rearrangement, consistent with a BRD4-NUT fusion and NMC. NUT carcinomas lacking BRD4 fusion rearrangements are more differentiated and, therefore, possibly less aggressive [11].

There is no recommended curative treatment for NMC. Many patients are treated with multimodal therapy (surgery, radiotherapy, and chemotherapy). The average life expectancy is 6-7 months and the overall survival is $19 \%$ at 2 years. However, a systematic review of all previously reported salivary gland NUT carcinomas $(n=15)$ showed the median survival as 24 and 4 months for pediatric and adult patients, respectively. The 1-year overall survival was $67 \%$ for pediatric and $11 \%$ for adult patients [12]. Yet, no chemotherapeutic agent has been discovered that extends the lifetime. Sopfe et al. reported a metastatic NUT midline carcinoma presenting as a wide metastasis in a patient who was in remission for 37 months after multimodal therapy with aggressive neoadjuvant chemotherapy, radiation, and resection [17]. Our patient showed a rapid progress with resistance to radiotherapy and chemotherapy, a month after the initial diagnosis, and died at 7 months after the diagnosis.

\section{Conclusion}

NMC, an aggressive tumor with nonglandular differentiation has to be interpreted in the differential diagnosis of poorly differentiated carcinomas. Various diagnostic methods for NUT protein, including antibodies, can be helpful. We would like to draw attention to the diagnostic challenge of this rare and highly aggressive carcinoma. The primary lesson of this case report is that NMC, which should be considered in the differential diagnosis of patients of any age presenting with "undifferentiated carcinoma" with midline localization, in addition to immunostaining for NUT, which can be characterized by FISH. NUT carcinoma has a dismal prognosis despite the aggressive multimodality management (surgery and adjuvant chemotherapy and/or radiation). New strategies are needed to improve outcomes of patients with this tumor. 
Author contributions. BYB substantially contributed to the conception and design of the work; BYB, DKY, UD, FÇ, YA, and $\mathrm{CAF}$ contributed to the acquisition of data. BYB, DKY, and UD analyzed and interpreted all data. BYB drafted the manuscript; and DKY and CAF revised it critically for important intellectual content. All authors approved the final version submitted for publication and agreed to be accountable for all aspects of the study in ensuring that questions related to the accuracy or integrity of any part of the study are appropriately investigated and resolved.

Acknowledgments. The authors thank Mr. Kaan Yildiz for assistance in preparing the manuscript. The material contained in this manuscript has been presented in a poster at the 24 ${ }^{\text {th }}$ National Pathology Congress, 2014 November 19-23, Trabzon, Turkey.

Conflicts of interest statement. The authors have each completed the International Committee of Medical Journal Editors Form for uniform Disclosure of Potential Conflicts of Interest. No authors have any potential conflict of interest to disclose.

Data sharing statement. All data generated or analyzed during the present study are included in this published article. Further details are available from the corresponding author on reasonable request.

\section{References}

[1] Schwartz BE, Hofer MD, Lemieux ME, Bauer DE, Cameron MJ, West NH, et al. Differentiation of NUT midline carcinoma by epigenomic reprogramming. Cancer Res. 2011; 71:2686-96.

[2] French CA, Ramirez CL, Kolmakova J, Hickman TT, Cameron MJ, Thyne ME, et al. BRD-NUT oncoproteins: a family of closely related nuclear proteins that block epithelial differentiation and maintain the growth of carcinoma cells. Oncogene. 2008; 27:2237-42.

[3] French CA. Pathogenesis of NUT midline carcinoma. Ann Rev Pathol. 2012; 7:247-65.

[4] Agaimy A, Fonseca I, Martins C, Thway K, Barrette R, Harrington KJ, et al. NUT Carcinoma of the salivary glands: clinicopathologic and molecular analysis of 3 cases and a survey of NUT expression in salivary gland carcinomas. Am J Surg Pathol. 2018; 42:877-84.
[5] Kees UR, Mulcahy MT, Willoughby ML. Intrathoracic carcinoma in an 11-year-old girl showing a translocation $\mathrm{t}(15 ; 19)$. Am J Pediatr Hematol Oncol. 1991; 13:459-64.

[6] Kubonishi I, Takehara N, Iwata J, Sonobe H, Ohtsuki Y, Abe T, Miyoshi I. Novel $\mathrm{t}(15 ; 19)(\mathrm{q} 15 ; \mathrm{p} 13)$ chromosome abnormality in a thymic carcinoma. Cancer Res. 1991; 51:3327-8.

[7] Stelow EB, Bellizzi AM, Taneja K, Mills SE, Legallo RD, Kutok JL, et al. NUT rearrangement in undifferentiated carcinomas of the upper aerodigestive tract. Am J Surg Pathol. 2008; 32:828-34.

[8] Bauer DE, Mitchell CM, Strait KM, Lathan CS, Stelow EB, Lüer SC, et al. Clinicopathologic features and long-term outcomes of NUT midline carcinoma. Clin Cancer Res. 2012; 18:5773-9.

[9] Chirieac LR, French CA, Sholl L, Yatabi Y. Lung: other and unclassified carcinomas. In: Travis WD, Brambilla E, Burke AP, Marx A, Nicholson AG, editors. WHO Classification of tumours of the lung, pleura, thymus and heart. 4th ed. Lyon: IARC Press; 2015. p. $97-8$.

[10] Boleto G, Perotin JM, Launois C, Uro-Coste E, Birembaut P, Dury S, et al. Nuclear protein in testis carcinoma of the mediastinum: a case report. J Med Case Rep. 2017; 11:152. doi: 10.1186/s13256017-1328-x

[11] Haack H, Johnson LA, Fry CJ, Crosby K, Polakiewicz RD, Stelow EB, et al. Diagnosis of NUT midline carcinoma using a NUT-specific monoclonal antibody. Am J Surg Pathol. 2009; 33:984-91.

[12] Wang H, Weiss VL, Hoffman RD, Abel T, Ho RH, Borinstein SC, et al. Salivary gland NUT carcinoma with prolonged survival in children: case illustration and systematic review of literature. Head Neck Pathol. 2020. doi: 10.1007/s12105-020-01141-3

[13] Prasad M, Baheti A, Ramadwar M, Chinnaswamy G, Vora T, Qureshi S. Pediatric NUT carcinoma is a rare and challenging tumor: single center experience of five children. Oncologist. 2019; 24:e1232-5.

[14] Lemelle L, Pierron G, Fréneaux P, Sophie Huybrechts S, Spiegel A, Plantaz D, et al. NUT carcinoma in children and adults: a multicenter retrospective study. Pediatr Blood Cancer. 2017; 64:e26693. doi: 10.1002/pbc.26693

[15] Zhou L, Yong X, Zhou J, Xu J, Wang C. Clinicopathological analysis of five cases of NUT midline carcinoma, including one with the gingiva. Biomed Res Int. 2020; 2020:9791208. doi: 10.1155/2020/9791208.

[16] Chau NG, Hurwitz S, Mitchell CM, Aserlind A, Grunfeld N, Kaplan L, et al. Intensive treatment and survival outcomes in NUT midline carcinoma of the head and neck. Cancer. 2016; 122:3632-40.

[17] Sopfe J, Greffe B, Treece AL. Metastatic NUT midline carcinoma treated with aggressive neoadjuvant chemotherapy, radiation, and resection: a case report and review of the literature. J Pediatr Hematol Oncol. 2020. doi: 10.1097/MPH.0000000000001860 